\title{
Phase retrieval based on diffraction element array with single far field (Withdrawal Notice)
}

Shuai Wang, Ping Yang, Lizhi Dong, Bing Xu, Mingwu Ao

Shuai Wang, Ping Yang, Lizhi Dong, Bing Xu, Mingwu Ao, "Phase retrieval based on diffraction element array with single far field (Withdrawal Notice)," Proc. SPIE 9526, Modeling Aspects in Optical Metrology V, 952613 (21 June 2015); doi: $10.1117 / 12.2184798$

SPIE. Event: SPIE Optical Metrology, 2015, Munich, Germany 


\section{Phase retrieval based on diffraction element array with single far field (Withdrawal Notice)}

Publisher's Note: This paper, originally published on June 21, 2015, was withdrawn at the author's request on July 7, 2015. 